EXTENDED REPORT

\title{
Autoantibodies to a NR2A peptide of the glutamate/ NMDA receptor in sera of patients with systemic lupus erythematosus
}

\author{
E S Husebye, Z M Sthoeger, M Dayan, H Zinger, D Elbirt, M Levite, E Mozes
}

See end of article for authors' affiliations

\section{Correspondence to:}

Professor Edna Mozes,

Department of

Immunology, The

Weizmann Institute of

Science, Rehovot 76100

Israel; edna.mozes@

weizmann.ac.il

Accepted 30 January 2005

Published Online first

11 February 2005

Objective: To determine the prevalence of autoantibodies directed against an epitope of the glutamate/ $\mathrm{N}$ methyl-D-aspartic acid (NMDA) receptor subunit NR2A (which is highly expressed in human brain) in the sera of lupus patients, and to investigate the possible correlation of these antibodies with clinical and serological manifestations of systemic lupus erythematosus (SLE).

Methods: Sera were obtained from 109 consecutive SLE patients. Controls were 65 patients with myasthenia gravis, 19 with autoimmune polyendocrine syndrome type I (APS I), and 65 healthy donors. A 15 amino acid long peptide based on a sequence within the NR2A subunit of the NMDA/glutamate receptor was synthesised. Antibodies to this peptide were determined by enzyme linked immunosorbent assay. Antibodies against double stranded DNA (dsDNA) were measured by Chrithidia luciliae assay. Disease activity was determined using the SLE disease activity index (SLEDAI).

Results: Sera of 34/109 SLE patients (31\%) reacted specifically with the NR2A peptide compared with only $4 / 65$ myasthenia gravis patients $(6.1 \%, p<0.001), 1 / 19$ APS I patients $(5.3 \%, p<0.02)$, and $3 / 65$ healthy controls $(4.6 \%, p<0.001)$. No correlation was found between the presence of NR2A and dsDNA or anti-cardiolipin specific autoantibodies. In addition, no significant correlation was observed between the presence of NR2A specific antibodies and the SLEDAI score or any lupus related clinical manifestations.

Conclusions: A significant number of SLE patients (31\%) have NR2A specific antibodies that do not correlate with anti-dsDNA antibodies. Additional studies of lupus patients with neurological disorders should elucidate the role of NR2A specific antibodies in lupus related CNS manifestations.

S ystemic lupus erythematosus (SLE) is an autoimmune disease characterised by impairment of $\mathrm{B}$ and $\mathrm{T}$ cell functions, cytokine dysregulation, and immune complex depositions accompanied by systemic clinical manifestations. ${ }^{12}$ The hallmark of SLE is the presence of a variety of autoantibodies directed mostly to double stranded DNA (dsDNA), nuclear antigens, ribonucleoproteins, and cell surface antigens. ${ }^{1-3}$ Some lupus associated clinical manifestations appear to be related to or mediated by specific autoantibodies (for example, anti-dsDNA and lupus nephritis $^{4}$; anticardiolipin antibodies and antiphospholipid syndrome $^{5}$ ). However, the mechanisms involved in the pathogenesis of SLE and the nature of the autoantigens are not yet defined.

About $10 \%$ of lupus patients have central nervous system (CNS) involvement, namely, psychosis or focal seizures. ${ }^{67}$ Moreover, non-focal neuropsychiatric disturbances including headache and cognitive decline are observed in up to $50 \%$ of the patients during the course of their disease..$^{78}$ The mechanism of these impairments is unknown, yet several studies have reported a correlation between lupus related neurological disorders and the presence of various autoantibodies directed against ribosomal P-protein, ${ }^{9}$ neurofilaments, ${ }^{10}$ or neuronal cells. ${ }^{11}$ Recently, DeGiorgio et al ${ }^{12}$ showed that a subset of anti-DNA antibodies (mouse monoclonal R4A antibodies and sera of some lupus patients) cross react with a sequence within the glutamate/NMDA receptor subunit NR2. Furthermore, injection of the cerebrospinal fluid of a patient with SLE and progressive neurological decline, which contained such cross reactive anti-DNA antibodies, into mouse brain caused neuronal death. ${ }^{12}$ These results suggested that cross reactive antibodies which recognise a common pentapeptide Asp/Glu-Trp-Asp/Glu-TyrSer/Gly, present in both dsDNA and N-methyl-D-aspartic acid (NMDA) receptor subunits NR2A and NR2B, may mediate lupus related disorders of the CNS.

The NMDA receptors, which bind the neurotransmitter glutamate and are present on neuronal cells throughout the brain, have been reported to play a role in many neurological functions, including learning and memory. ${ }^{13}$ As other studies have reported the presence of NMDA receptors on nonneuronal cells, including platelets, ${ }^{14}$ autoantibodies directed against these receptors may also be involved in the pathogenesis of other (non-neurological) SLE related clinical manifestations.

The present study was conducted to determine the prevalence of antibodies against an epitope of the glutamate/NMDA receptor subunit NR2A in the sera of a large cohort of lupus patients, to define the correlation of the latter antibodies with dsDNA specific antibodies, and to investigate the possible correlation between the presence of the NR2A specific antibodies and SLE related clinical manifestations and disease activity.

\section{METHODS}

\section{Patients}

One hundred and nine patients (16 men, 93 women) with SLE participated in the study. All patients had at least four of

Abbreviations: AMPA, 2-amino-3-(3-hydroxy-5-methylisoxazol-4-yl); ANA, anti nuclear antibodies; APS I, autoimmune polyendocrine syndrome type I; dsDNA, double stranded deoxyribonucleic acid; MG, myasthenia gravis; NMDA, N-methyl-D-aspartic acid; SLE, systemic lupus erythematosus; SLEDAI, SLE disease activity index 


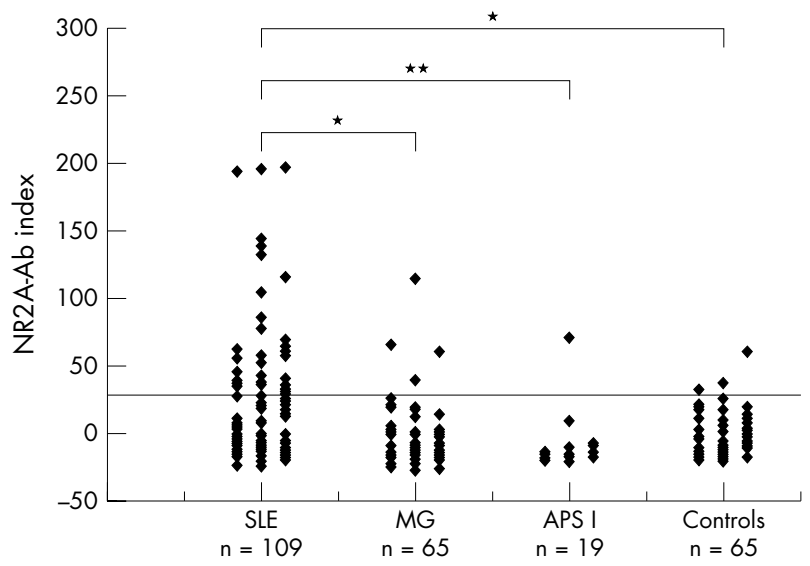

Figure 1 Antibodies against the NR2A peptide of the glutamate/ NMDA receptor in sera of patients with systemic lupus erythematosus (SLE). Sera from 109 SLE patients, 65 patients with myasthenia gravis (MG), 19 patients with autoimmune polyendocrine syndrome type I (APS1), and 65 healthy controls were tested. NR2A specific antibodies were determined by enzyme linked immunosorbent assay (ELISA). Results are expressed as NR2A antibody indices, calculated as described in Methods. The horizontal line represents the upper normal limit (NR2A antibody index of 30) defined as the mean of 65 normal controls +2 SD. ${ }^{*} \mathrm{p}<0.001,{ }^{* *} \mathrm{p}<0.02$.

the American College of Rheumatology revised classification criteria for SLE. ${ }^{15}$ The mean (SD) age at diagnosis was 34 (13.1) years and the mean follow up period was 14 (10.8) years. All patients had antinuclear antibodies (ANA) in their sera (tested at 1:100 dilution) as determined by a Hep2 cell assay. ${ }^{16}$ Eighty three per cent of the patients had dsDNA specific reactivity as measured (at sera dilution of $1: 10$ ) by Chrithidia luciliae assay ${ }^{16}$ at least at one time point during the follow up period. The results of dsDNA reactivity are presented as dsDNA scores (negative, +1 to +4 scores). Antiphospholipid antibodies-determined by the presence of anticardiolipin antibodies or lupus anticoagulants, or bothwere detected in $28 \%$ of the patients. Arthritis was observed in $85 / 109$ patients $(78 \%)$. Haematological involvement (haemolytic anaemia, thrombocytopenia, leucopenia, lymphopenia, or combinations of these) and renal involvement were observed in $68 \%$ and $38 \%$ of the patients, respectively. Neurological manifestations, defined in the present study as psychosis or focal seizures, were observed in six of the patients $(5.5 \%)$ during the follow up period. SLE disease activity was determined using the SLEDAI lupus activity index. ${ }^{17}$ The mean (SD) SLEDAI score at the time of the study was 6.32 (5.13) (range 0 to 25).

Fifty per cent of the patients were treated with steroids at the time of the study and 15\% were treated with cytotoxic agents (either cyclophosphamide, azathioprine, or methotrexate).

As controls we studied sera from 65 patients with myasthenia gravis. Although myasthenia gravis is a neurological disease involving the neuromuscular junction rather than the brain, we chose it as a control condition because of its autoimmune nature. In addition, 19 patients with autoimmune polyendocrine syndrome type I (APS I) and 65 age and sex matched healthy controls were tested.

All participants signed an informed consent form before initiation of the study, which was approved by the ethics committee of the Kaplan Medical Centre.

\section{Synthetic peptide}

The 15 amino acid peptide SVSYDDWDYSLEARV (amino acids 278 to 292) of the NR2A subunit of the NMDA subtype

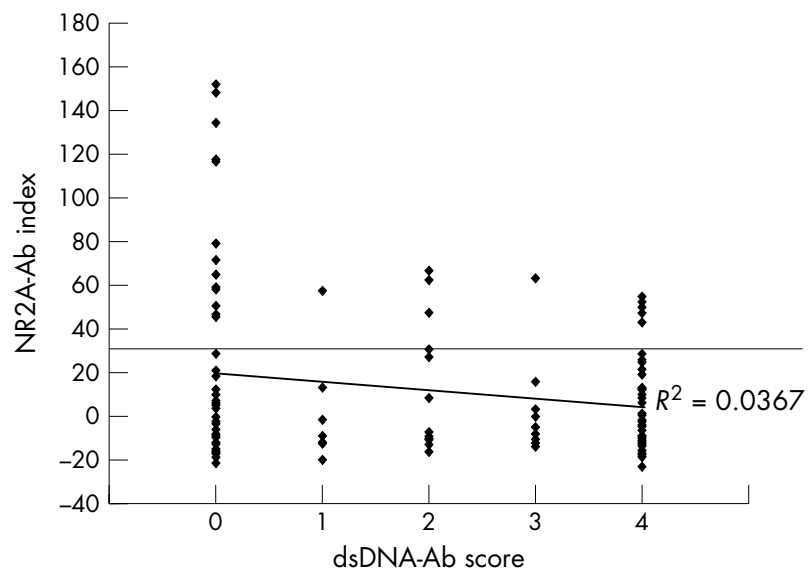

Figure 2 Correlation between NR2A antibody indices and dsDNA reactivity in sera of patients with systemic lupus erythematosus (SLE). The NR2A antibody indices and dsDNA antibody reactivity (negative to +4 ) were compared in 125 serum samples from 59 SLE patients (two or more serum samples per patient). The line represents the linear regression. dsDNA, double stranded deoxyribonucleic acid.

of the glutamate receptor that contains the DWDYS epitope ${ }^{12}$ was synthesised with an automated synthesiser (Applied Biosystems model 430A) using the company's protocol for t-butyloxycarbonyl strategy. ${ }^{18}$

\section{Evaluation of antibodies against the NR2A peptide} Antibodies against the NR2A peptide were measured by enzyme linked immunosorbent assay (ELISA) using 96-well microtitre plates (Nunc, Denmark). The plates were coated with $0.025 \mu \mathrm{g}$ peptide per well at $4^{\circ} \mathrm{C}$ overnight. They were washed four times with phosphate buffered saline containing 0.05\% Tween-20 (PBS-T) (Sigma, St Louis, Missouri, USA). Blocking was undertaken with PBS containing 10\% milk with $1 \%$ fat for two hours at room temperature. After washing with PBS-T, plates were incubated with sera dilutions ( $1: 50)$ for three hours at room temperature. Following further washing, peroxidase conjugated goat antihuman IgG was added at a 1:5000 dilution. Plates were developed using 2,2'azino-bis (3-ethylbenzthiazoline-6-sulphonic acid) (Sigma) and read after 30 minutes at $405 \mathrm{~nm}$ by an ELISA reader. In each assay a pool of 42 normal control sera was used as a routine standard negative control and a serum found in the initial ELISA to bind strongly to NR2A was used in all subsequent assays as a positive control. The positive and negative controls were tested in quadruplicate on each plate. The results were expressed as an NR2A index calculated as follows: [optical density (OD) of sample - OD negative control] / [OD positive control - OD negative control] $\times 100$. The mean of the 65 normal controls +2 SD was used as the upper limit of normal. The intra-assay and interassay variability was $5 \%$ and $15 \%$, respectively.

\section{Statistical analyses}

The results are presented as mean (SD). The linear regression $\left(R^{2}\right)$ test (using Microsoft Excel), $\chi^{2}$, Student's $t$ tests were used for statistical analyses. A probability $(p)$ value of $\leqslant 0.05$ was considered significant.

\section{RESULTS}

\section{NR2A specific antibodies in sera of SLE patients}

In order to assess the frequency of antibodies reactive with the NR2A peptide in the sera of SLE patients, sera from 109 consecutive lupus patients, 65 patients with myasthenia gravis, 19 patients with APS I, and 65 healthy donors were 

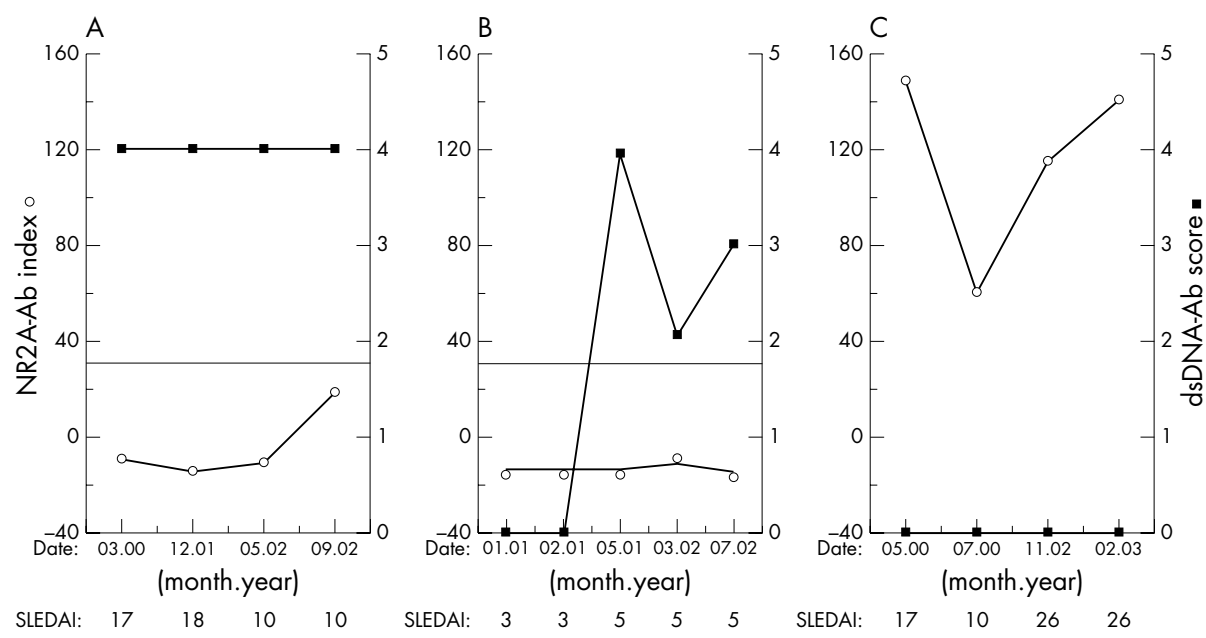

Figure 3 Correlation between NR2A antibody indices, dsDNA specific antibodies, and systemic lupus erythematosus disease activity index (SLEDAI). NR2A antibody index, dsDNA reactivity, and SLEDAl were determined in three different SLE patients $(A, B, C)$ during a follow up period of 18 to 33 months. dsDNA, double stranded deoxyribonucleic acid.

tested (at 1:50 dilution) in an NR2A specific ELISA. Figure 1 shows that $34 / 109(31 \%)$ of lupus patients reacted specifically with the NR2A peptide, compared with only $4 / 65$ myasthenia gravis patients $(6.1 \%, \mathrm{p}<0.001), \mathrm{l} / 19$ APS I patients $(5.3 \%$, $\mathrm{p}<0.02)$, and $3 / 65$ healthy controls $(4.6 \%, \mathrm{p}<0.001)$.

\section{Relation between NR2A and dsDNA specific autoantibodies in sera of SLE patients}

To investigate the possible relation between NR2A and dsDNA specific autoantibodies we evaluated concomitantly the reactivity to NR2A and dsDNA in sera obtained from 59 of our SLE patients, in whom two or more serum samples drawn at different time points were available for evaluation. As shown in fig 2, no correlation was found between dsDNA reactivity at different time points along the study and the presence of or levels of NR2A specific antibodies. Moreover, sera with the highest reactivity of NR2A specific antibodies (NR2A index $>80$ ) were negative for dsDNA antibodies, whereas most of the sera which showed high dsDNA reactivity (defined as dsDNA score of +4 ) did not react significantly with the NR2A peptide (fig $2 ; R^{2}=0.037$ ). Analysis based on one serum sample of each patient also did not show any correlation between NR2A and dsDNA specific antibodies $\left(R^{2}=0.0006\right)$. In addition, there was no correlation between the levels of antiphospholipid antibodies (anticardiolipin or lupus anticoagulant or both) and NR2A specific antibodies $\left(R^{2}=0.0078\right)$. Similarly, the levels of ANA measured on Hep2 cells did not correlate with the magnitude of NR2A activity $\left(R^{2}=0.0008\right)$. The relations between NR2A and dsDNA specific antibodies were further evaluated in three individual patients at different time points (fig 3). As can be seen in fig $3 \mathrm{~A}$, sera from patient 1 did not react significantly with the NR2A peptide but had high titres $(+4$ score) of dsDNA specific antibodies at all time points during a 2.5 year follow up period. Patient 2 also had no NR2A specific antibody reactivity in his sera whereas the anti-dsDNA antibody levels varied (between scores of 0 to +4 ) during the 18 months of the follow up (fig 3B). Finally, patient 3 had high levels of NR2A specific antibodies but no dsDNA autoantibodies during a follow up period of about three years (fig 3C).

\section{Relation between NR2A specific antibodies and clinical manifestations of SLE}

Neither the lupus related clinical manifestations (neurological, renal, haematological, mucocutaneous, and musculoskeletal involvement) nor the SLEDAI correlated significantly with the presence of or titres of NR2A antibodies in the patients' sera $\left(R^{2}=0.0238\right)$. Treatment with steroids or cytotoxic drugs did not affect the presence of or titres of the anti-NR2A antibodies.

It is noteworthy that the small number of patients $(6 / 109$, $5.5 \%$ ) with neurological involvement, defined as psychosis or seizure, limited the ability to determine the possible association and the role of the NR2A antibodies in the development of lupus related CNS disease. Although no significant correlation was observed between the presence of NR2A antibodies and lupus related CNS involvement, the two highest NR2A antibody indices were detected in sera from patients with CNS manifestations. Furthermore, fig 3C shows high NR2A specific antibody levels in the sera of a patient with severe neurological manifestations. In this particular SLE patient, who suffered from organic brain syndrome, cranial nerve disorders, and visual disturbances, the titres of NR2A specific antibodies correlated with the severity of the neurological involvement. Thus the clinical improvement of her neurological symptoms (July 2000) was associated with a significant decrease in the NR2A antibody index (fig 3C). When neurological manifestations worsened again (November 2002), the NR2A antibody index also increased (fig 3C). During this period no anti-dsDNA antibodies were detected in serum samples from this patient. Nevertheless, further studies of SLE patients with well defined neuropsychiatric lupus manifestations ${ }^{19}$ should determine the role of the NR2A specific antibodies in neurological lupus.

\section{DISCUSSION}

The main finding of the present study was that a significant number of SLE patients $(34 / 109=31 \%)$ have raised levels of antibodies in their sera directed against a peptide consisting of amino acids 278-292 of the NR2A subunit of the NMDA subtype of glutamate receptors-that is, anti-NR2A antibodies. The specific association of the NR2A antibodies with SLE is supported by the low frequency of these antibodies in sera from patients with myasthenia gravis (4/65), APS I ( $1 /$ 19 ), or healthy controls (3/65) shown in this study (fig 1 ).

Glutamate is the major excitatory neurotransmitter of the nervous system and plays a crucial role in a variety of key functions in the central nervous system, including learning and memory. ${ }^{13}$ Glutamate has ionotropic (ion channel) receptors and G-protein coupled metabotropic receptors. ${ }^{20}$ The ionotropic glutamate receptors are further subdivided into the 2-amino-3-(3-hydroxy-5-methylisoxazol-4-yl) propionic acid (AMPA)/kainate receptors and the NMDA receptors, based on their preferential affinities for the synthetic excitatory NMDA or AMPA, respectively. ${ }^{20} 21$ 
Molecular cloning has revealed three receptor subunit families (NR1, NR2, and NR3A) which form hetero-oligomeric complexes in native NMDA receptor channels. ${ }^{21}$ NMDA receptors are of major interest as they are involved in many processes necessary for brain development, including neuronal migration, ${ }^{20}$ patterning of afferent termination, ${ }^{21}$ and several forms of long term synaptic plasticity. ${ }^{22}$ In addition, NMDA receptors have also been implicated as a mediator of neuronal injury caused by excess glutamate (excitotoxicity) and taking place in many neurological disorders including stroke, epilepsy, brain trauma, dementia, and neurodegenerative disorders. ${ }^{23}{ }^{24}$ Thus in principle antibodies specific to the NMDA receptors may lead to neurological disturbances.

Antibodies against the NMDA receptor subunit families have been found in the sera of patients with nonautoimmune neurological disorders. Thus antibodies to NR2B were recently found in sera from 13 of 15 patients with chronic forms of epilepsy. ${ }^{25}$ Anti-NR2 (NR2A/2B) antibodies were also reported in sera from patients with ischaemic stroke (94\%), including transient ischaemic attacks $(75 \%) .^{22}$ We recently found anti-NR2A specific antibodies (amino acids 278-292) in 20\% of patients with different types of epilepsy (Ganor et al, unpublished data). DeGiorgio $\mathrm{et}_{\mathrm{al}}{ }^{12}$ found neuronal damage upon injection into mice of anti-NR2 glutamate receptor binding antibodies contained in two sera and one CSF sample from SLE patients. We report here the presence of NR2A specific antibodies in $31 \%$ of the sera of a large cohort of SLE patients (fig 1).

The findings of the present study suggest that the antiNR2A antibodies are distinct from anti-dsDNA autoantibodies, which are the hallmark of SLE. Although we have not undertaken absorption experiments, the latter suggestion is based on the lack of association between the titres of antibodies directed to dsDNA and to NR2A (figs 2 and 3). In fact, the results showed that patients with the highest dsDNA specific antibody titres at the time of the test had the lowest NR2A specific antibody indices and vice versa, raising the possibility of a negative correlation between these antibodies.

It is noteworthy that DeGiorgio et $a l^{12}$ reported that a mouse monoclonal (IgG2b, R4A) anti-DNA antibody and a limited number of human SLE sera with dsDNA specific antibodies cross reacted with the NR2A and NR2B subunits of the NMDA receptor. It appears that the anti-NR2A antibodies found herein differ from the NR2 binding of the anti-dsDNA IgG2b R4A monoclonal antibody shown in the latter study ${ }^{12}$. It is also likely that because of the different approaches of the two studies different subsets of NR2A specific antibodies were investigated. Nevertheless, although we cannot rule out cross reactivity between dsDNA and NR2A in a very limited number of sera, the NR2A specific antibodies that were detected in the sera of a significant number of SLE patients studied here (fig 1) did not correlate with anti-dsDNA reactivity (figs 2 and 3 ).

\section{Conclusion}

A significant number (31\%) of SLE patients have raised levels of antibodies to the NR2A peptide of the glutamate/NMDA receptor. Our findings call for further studies of SLE patients with various neurological and cognitive manifestations ${ }^{19}$ to explore the potential contribution of the NR2A specific antibodies to the lupus related CNS involvement.

\section{ACKNOWLEDGEMENTS}

The work was supported by Teva Pharmaceutical Industries Limited, Israel. ESH was supported by grants from the Norwegian Research Council and the Weston Scholarship Program of The Weizmann Institute of Science. We thank Professor Vivian Teichberg (WIS) for enlightening discussions on the structure and function of NMDA receptors in health and disease.

\section{Authors' affiliations}

E S Husebye*, M Dayan, H Zinger, E Mozes, Department of Immunology, Weizmann Institute of Science, Rehovot, Israel

$M$ Levite, Department of Neurobiology, Weizmann Institute of Science Z M Sthoeger, D Elbirt, Department of Medicine " $B$ ", Kaplan Hospital, Rehovot, Israel

*Present address: Division of Endocrinology, Institute of Medicine, Haukeland University Hospital, Bergen, Norway.

\section{REFERENCES}

1 Hahn BH. An overview of the pathogenesis of systemic lupus erythematosus. In: Wallace DJ, Hahn BH, eds. Dubois' lupus erythematosus. Philadelphia: Williams and Wilkins, 1993:69-76.

2 Winchester RJ. Systemic lupus erythematosus pathogenesis. In: Koopman W, ed. Arthritis and allied conditions. Birmingham, Alabama: Williams and Wilkins, 1996:1361-91.

3 Arbuckle MR, McClain MT, Rubertone MV, Scofield RH, Dennis GJ, James JA, et al. Development of autoantibodies before the clinical onset of systemic lupus erythematosus. N Engl J Med 2003;349:1526-33.

4 Lefkowith JB, Gilkeson GS. Nephritogenic autoantibodies in lupus: current concepts and continuing controversies. Arthritis Rheum 1996:39:894-903.

5 Sthoeger ZM, Tartakovsky B, Bentwich Z, Mozes E. Monoclonal anticardiolipin antibodies derived from mice with experimental lupus erythematosus: characterization and the induction of a secondary antiphospholipid syndrome. J Clin Immunol 1993;13:127-38.

6 Ainiala H, Loukkola J, Peltola J, Korpela M, Hietaharju A. The prevalence of neuropsychiatric syndromes in systemic lupus erythematosus. Neurology 2001;57:496-500.

7 Sanna G, Bertolaccini ML, Cuadrado MJ, Laing H, Khamashta MA, Mathieu A, et al. Neuropsychiatric manifestations in systemic lupus erythematosus: prevalence and association with antiphospholipid antibodies. J Rheumatol 2003;30:985-92.

8 Loukkola J, Laine M, Ainiala H, Peltola J, Metsanoja R, Auvinen A, et al. Cognitive impairment in systemic lupus erythematosus and neuropsychiatric systemic lupus erythematosus: a population-based neuropsychological study. J Clin Exp Neuropsychol 2003;25:145-51.

9 Reichlin M. Ribosomal P antibodies and CNS lupus. Lupus 2003;12:916-18.

10 Kurki P, Helve T, Dahl D, Virtanen I. Neurofilament antibodies in systemic lupus erythematosus. J Rheumatol 1986;13:69-73.

11 Williams RC, Sugiura K, Tan EM. Antibodies to microtubule-associated protein 2 in patients with neuropsychiatric systemic lupus erythematosus. Arthritis Rheum 2004;50:1239-47.

12 DeGiorgio LA, Konstantinov KN, Lee SC, Hardin JA, Volpe BT. Diamond B. A subset of lupus anti-DNA antibodies cross-reacts with the NR2 glutamate receptor in systemic lupus erythematosus. Nat Med 2001;7:1 189-93.

13 Morris RG, Anderson E, Lynch GS, Baudry M. Selective impairment of learning and blockade of long-term potentiation by an N-methyl-D-aspartate receptor antagonist, AP5. Nature 1986;319:774-6.

14 Skerry TM, Genever PG. Glutamate signalling in non-neuronal tissues. Trends Pharmacol Sci $2001 ; 22: 174-81$.

15 Tan EM, Cohen AS, Fries JF, Masi AT, McShane DJ, Rothfield NF, et al. The 1982 revised criteria for the classification of systemic lupus erythematosus. Arthritis Rheum 1982;25:1271-7.

16 Tan EM. Autoantibodies to nuclear antigens (ANA): their biology and medicine. Adv Immunol 1982;33:167-240.

17 Bombardier C, Gladman DD, Urowitz MB, Caron D, Chang CH. Derivation of the SLEDAI. A disease activity index for lupus patients. The Committee on Prognosis Studies in SLE. Arthritis Rheum 1992;35:630-40.

18 Schnolzer M, Kent SB. Constructing proteins by dovetailing unprotected synthetic peptides: backbone-engineered HIV protease. Science 1992;256:221-5.

19 ACR Ad Hoc Committee on Neuropsychiatric Lupus Nomenclature. The American College of Rheumatology nomenclature and case definitions for neuropsychiatric lupus syndromes. Arthritis Rheum 1999;42:599-608.

20 Mayer ML, Armstrong N. Structure and functional of glutamate receptor ion channels. Annu Rev Physiol 2004;66:161-81.

21 Scatton B. The NMDA receptor complex. Fundam Clin Pharmacol 1993;7:389-400.

22 Dambinova SA, Khounteev GA, Izykenova GA, Zavolokov IG, llyukhina AY, Skoromets AA. Blood test detecting autoantibodies to N-methyl-D-aspartate neuroreceptors for evaluation of patients with transient ischemic attack and stroke. Clin Chem 2003;49:1752-62

23 Choi DW, Rothman SM. The role of glutamate neurotoxicity in hypoxicischemic neuronal death. Annu Rev Neurosci 1990;13:171-82.

24 Mattson MP, LaFerla FM, Chan SL, Leissring MA, Shepel PN, Geiger JD. Calcium signaling in the ER: its role in neuronal plasticity and neurodegenerative disorders. Trends Neurosci 2000;23:222-9.

25 Takahashi Y, Mori H, Mishina M, Watanabe M, Fujiwara T, Shimomura J, Aiba $\mathrm{H}$. Autoantibodies to NMDA receptor in patients with chronic forms of epilepsia partialis continua. Neurology 2003;61:891-6. 